\title{
BIOÉTICA EN LA ERA DEL COVID-19
}

\author{
Sánchez , Ana \\ Miembro principal del Comité Nacional de Bioética de la Investigación \\ Asesora de la Secretaría Nacional de Ciencia Y Tecnología e Innovación (SENACYT) \\ Especialista en Bioética \\ Panamá, Panamá \\ asanchez@senacyt.gob.pa \\ Casado, María \\ Fundadora del Observatorio de Bioética y Derecho de la Universidad de Barcelona \\ Directora del Máster en Bioética y Derecho de la Universidad de Barcelona \\ Catedrática Acreditada de Filosofía del Derecho \\ Barcelona, España \\ mariacasado@ub.edu ORCID: 0000-0003-1586-9502

\section{Saénz , Carla} \\ Asesora Regional de Bioética de la Organización Panamericana de la Salud-OPS \\ Departamento de Sistemas y Servicios de Salud de la OPS \\ Responsable del Programa Regional de Bioética de la OPS \\ Washington, D.C. United States \\ saenzcar@paho.org

\section{Ying , Argentina} \\ Presidenta del Comité Nacional de Bioética de la Investigación (CNBI) \\ Representante de la Universidad de Panamá en el CNBI \\ Miembro del Comité de Bioética de la Universidad de Panamá \\ Investigadora y Catedrática de la Facultad de Medicina de la Universidad de Panamá \\ Panamá, Panamá \\ Argentina.ying@up.ac.pa ORCID:0000-0001-7981-9139
}

\section{Abstract}

With the objective of making a discussion and reflection on Bioethics in the Covid 19 Pandemic, through a holistic vision, the point of view of an international technical cooperation advisory entity and the operationalization of the bioethical review of research in Central America and the Dominican Republic, we present this round table, moderated by Dr. Ana Sánchez. The Covid-19 Pandemic has impacted not only on the global health of human beings, it has also affected all aspects of daily life, exposing pre-existing inequalities 
and even increasing them. From an ethical perspective, María Casado brought to the table for discussion the impact of the Pandemic on human rights, the risks of producing a huge database as a result of an accelerated research, and the capacity of ethics committees to confront conflicts of interest. From the point of view of the Pan American Health Organization with Carla Saénz we reflected on how prepared we were to carry out an ethical review in emergency situations and disease outbreaks, what were the lessons learned and what perspectives we will have to face in future situations of emergency. Finally, Argentina Ying presented the situation of the ethical evaluation of research in Covid-19 in Central America and the Dominican Republic, highlighting the diversity of ethical governance situations in the region, the need to strengthen Bioethics in research and the importance of international cooperation to close or reduce existing gaps.

Keywords: Bioethics, Pandemic, governance

\section{Resumen}

Con el objetivo de hacer una discusión y reflexión sobre la Bioética en la Pandemia de la Covid 19, a través de una visión holística, el enfoque de una entidad internacional asesora de cooperación técnica y la operacionalización de la revisión bioética de la investigación en Centroamérica y República Dominicana, presentamos esta mesa redonda, moderada por la Dra. Ana Sánchez. La Pandemia Covid-19 ha impactado no solamente en la salud global de los seres humanos, también ha incidido en todos los aspectos de la vida cotidiana, desnudando las desigualdades preexistentes e incluso incrementándolas. Desde la perspectiva ética, María Casado se puso en la mesa de discusión el impacto de la Pandemia en los derechos humanos, los riesgos de la producción de una enorme base de datos producto de la investigación acelerada y la capacidad de los comités de ética para enfrentar los conflictos de interés. Desde el punto de vista de la Organización Panamericana de la Salud con Carla Saénz reflexionamos sobre cuán preparados estábamos para hacer una revisión ética en situaciones de emergencia y brotes de enfermedades, cuáles fueron las leciones aprendidas y qué perspectivas tendremos para enfrentar en el futuro situaciones de emergencia. Finalmente Argentina Ying presentó la situación de la evaluación ética de la investigación en la Covid-19 en Centroamérica y Republica Dominicana, resaltando la diversidad de situaciones de gobernanza ética en la región, la necesidad de fortalecer la Bioética en investigación y la importancia de la cooperación internacional para cerrar o disminuir las brechas existentes.

Palabras claves: Bioética, Pandemia, gobernanza 


\section{INTRODUCCIÓN}

Paralelo a la declaración de Pandemia en marzo de 2020, muchos países del mundo adoptaron medidas urgentes a fin de intentar frenar el rápido crecimiento del contagio de la Covid-19, medidas que incluyeron además del impedimento de circulación de las personas, restricción de derechos fundamentales debido a la existencia de un peligro eminente de salud pública. También se tomaron decisiones económicas, otorgando subsidios a la población que ha dejado de trabajar, moratorias en pago de deuda, y en ese sentido toda esa serie de movilizaciones anormales o no comunes en el funcionamiento de diversos estados.

Entre estos también se facilitaron incentivos específicos a la investigación científica, para enfrentar el tema de la pandemia creando un espacio para el análisis bioético que permitiera hacer frente a los conflictos que pueden derivar de la divergencia de opiniones, la pluralidad de intereses dentro de la población y las necesidades de protección de la colectividad de grupo o persona de manera individual. En este caso tratándose de una pandemia por enfermedad infecciosa fue necesario controlar la enfermedad y tomar decisiones urgentes y precipitadas, por lo que es necesario poner a debate la acción del estado, tomando en cuenta que no somos una unanimidad de opiniones, si no que hay divergencias y matices diversos que se tiene que tomar en cuenta.

\section{OBJETIVOS}

Poner en contexto las medidas adoptadas, sus aciertos y desaciertos tanto a nivel de la prevención de la enfermedad su impacto social, económico y humano.

Debatir si la medida para incentivar la investigación cumplió los objetivos propuestos.

\section{MATERIALES Y MÉTODOS}

Se realizó una mesa redonda para la presentación, del impacto ético de la Pandemia Covid -19 desde diferentes ámbitos. Con la participación de la Dra. María Casado, se planteó de manera integral el impacto de la Pandemia en la vida cotidiana, considerando en primer término la importancia de hacer un buen análisis, para poder tomar medidas acertadas. La Pandemia ha puesto de manifiesto no solo la crisis de salud, si no también la crisis económica y social, en una situación que aparentemente sería pasajera pero que no solo se ha alargado, sino que tampoco se acaba de ver el final completo del túnel en el que estamos. Durante la pandemia ha habido una explosión de la digitalización, y una explosión de un sistema de control al que nosotros no estábamos acostumbrados, por tanto, es muy importante todo lo que tenga que ver con el uso y abuso de los datos, toda la discusión sobre los sistemas de detección de casos y de contagios, el llamado radar Covid. Se recaudó una enorme cantidad de datos cuya disponibilidad sumada al potencial que 
tiene la inteligencia artificial van a impactar en la vida de las personas, en su control y en las decisiones que se van a tomar sobre ellas. Este es un tema extremadamente importante que ha calado en el ámbito de la bioética y en el ámbito de la vida de nuestros conciudadanos ya que son decisiones políticas que llevan detrás concesiones del mundo, concesiones de los derechos, concesiones de la dignidad, concesiones de la vida buena por lo que llamó la atención al tema conflicto de intereses de las personas que toman las decisiones que nos afectan a todos. Hay poco control de las investigaciones que se están haciendo, y de la privatización del beneficio que esto está generando, debemos darnos cuenta de que la realidad es que la pandemia no es solo es un problema de salud, sino que es un problema generalizado de ciudadanía y de clase global. Con respecto a la investigación en Covid-19 asombra la rapidez con que se han obtenido los resultados, el acortar los plazos no significa que debamos aligerar o hacer menos exigentes los procedimientos, no hay atajo en este sentido, si los ciudadanos pierden la confianza en la ciencia será muy difícil recuperarla. En este tiempo de investigación de la pandemia, se ha visto que el esfuerzo combinado ha dado muy buenos resultados, aunque paralelamente ha habido mucha investigación movida por la competencia, por llegar primero con conflictos de interés evidentes. Es preocupante que la integridad científica no se respete lo suficiente, tenemos que hacer mucho hincapié en estos temas de conflicto de intereses, de evitar la presión de hacer las cosas rápidas por publicarlas y hacerlas realmente bien. Hay que tener muy claro el papel de los comités de bioética y la capacitación de los miembros en temas como la digitalización, uso de los datos, inteligencia artificial y sobre todo conflicto de intereses. La bioética si algo puede aportar en estos momentos es intentar llamar a las cosas por su nombre, intentar hacer un análisis riguroso, analizar cómo han funcionado las cosas y determinar cuáles serían los cursos de acción que podemos emprender a partir de ahora, porque esta situación se va a mantener, y es evidente que no puede ser que sigamos en la misma línea de concentración de poder y recursos en las mismas manos y de exclusión de las otras.

Desde la perspectiva programa Regional de Bioética de la OPS Carla Sáenz expuso que es indiscutible que la Pandemia de Covid-19 ha puesto la Bioética en la palestra pública, todos están hablando de bioética, está claro que la pandemia nos enfrenta a desafíos éticos. En la región de las Américas, ha sido posible construir sobre la experiencia del brote del Zika, quedando claro que hacer investigación en emergencia sanitaria es un imperativo ético, no es un obstáculo, es una parte consustancial de la respuesta a la situación. Otra cosa que está clara es que toda investigación realizada en emergencia debe obtener aprobación ética previa. Igualmente, no hay dudas en cuanto a que los procesos de revisión ética (no los estándares éticos) deben ajustarse a la situación de emergencia, deben hacerse de manera más rápida acorde a la realidad, aunque reconocemos que iniciamos la pandemia sin tener claridad sobre cómo hacerlo exactamente. Un punto importante a considerar 
en donde la puesta en práctica esta en juego, tiene que ver con el volumen inusual de evidencias producidas en corto tiempo, ocurriendo que la evaluación de un estudio que tenía validez social y científica con un balance positivo de los beneficios frente a los riesgos, un mes mas tarde con la nueva evidencia disponible, ya no tiene valor social, o el diseño científico ya no es éticamente aceptable o el balance de riesgos beneficios se ha inclinado en sentido negativo. Otra reflexión temática, tiene que ver con las intervenciones de salud que impactan a las poblaciones, lo que llamamos desde el brote del Ébola el marco MEURI. Se ha encontrado que muchas intervenciones que no han sido previamente probadas para la Covid-19, se están ofreciendo para la Covid-19 fuera de un ámbito de investigación ¿Por qué esto es éticamente preocupante? En situaciones normales hacemos investigación, para probar si una intervención funciona o no, entonces hablamos de participante de investigación y aseguramos una serie de salvaguardas para aquellos participantes que están recibiendo una intervención no probada. Una vez que una intervención es probada y sabemos que es segura y eficaz se le brinda a la población fuera de un marco de investigación. El problema con el que nos hemos encontrado es que se están dando intervenciones no probadas aún, pero al darse fuera de un marco de investigación no se provee a las personas de la salvaguarda apropiada puesto que estamos hablando de intervenciones que podrían no beneficiar o incluso perjudicar. En la OPS se ha producido una orientación específica, denominada "Uso de emergencia de intervenciones no probadas y fuera del ámbito de la investigación: orientación ética para la pandemia de Covid-19", documento que sigue siendo al día de hoy la orientación ética de la organización. Estos temas sobre el uso de intervenciones no probadas rebasaron las discusiones de los profesionales de la salud, de los bioeticistas, los investigadores y son parte del discurso del publico en general. En repuesta a esta realidad, por primera vez en más de 10 años, como parte de la cooperación técnica de la OPS nos hemos dirigido directamente a las personas y hemos empezado a producir material de ética para el público en general, tendencia que llegó para quedarse y que tiene el lado positivo de abrir el ámbito de la reflexión ética a un público mas amplio. Finalmente acotó que hay un trabajo pendiente muy importante que hacer con nuestras autoridades sanitarias y que tiene que ver con especificar el rol de la ética en la toma de decisiones en situaciones en que hay un bajo umbral de evidencia o mucha incertidumbre. Esto hace obvio que hay que explicitar el análisis ético que justifica o no justifica cierta intervención en salud, un poco retomando una idea también de la Dra. Casado relativa a la necesidad de hacer más análisis ético, pero más análisis ético explícito, y explícito para la población en general.

Argentina Ying presentó la respuesta a la revisión ética de la investigación de acuerdo a los resultados de la investigación "Fortalecimiento de la gobernanza y Regulación ética de la investigación en América Central y República Dominicana en respuesta a la Pandemia 
de Covid-19 (GoÉtica); y la experiencia de Panamá, los desafíos, las lecciones aprendidas y los logros de la revisión ética de la investigación en la Pandemia de la Covid-19. Se trata de una investigación patrocinada por la Organización Mundial de la Salud, con la participación de Costa Rica, Honduras, El Salvador, Guatemala, Panamá y República Dominicana. En el Taller titulado "Desafíos de la regulación, la revisión ética y la vigilancia de la investigación en salud durante emergencias de salud pública" cuyos objetivos eran: Identificar las fortalezas, debilidades, desafíos y obstáculos de la revisión ética de la investigación durante emergencias de salud pública; e identificar estrategias viables que faciliten el trabajo coordinado de la revisión ética durante emergencias de salud pública a nivel nacional y regional; participaron 83 personas con representación de los 6 países del estudio y observadores de Perú y México, de los cuales el $69 \%$ era del genero femenino y $31 \%$ del género masculino.
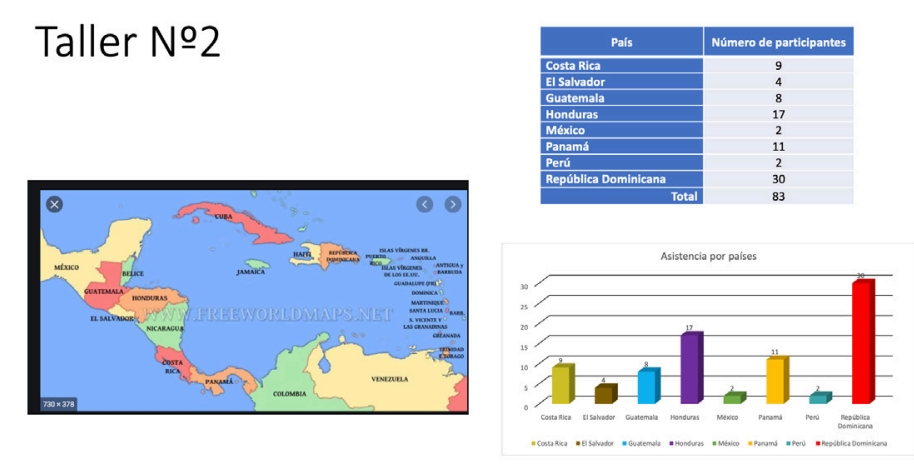

En cuanto a la diversidad o multidisciplinariedad de los participantes, hubo autoridades gubernamentales, docentes, estudiantes, bioeticistas (expertos) investigadores, miembros de comités de bioética y representantes de organizaciones de base.

En el cuadro №1 se presentan los resultados iniciando con la pregunta "Existe un documento escrito relativo a las prioridades de investigación en Covid-19", ninguno de los países tenía un documento específico, solo dos países Panamá y El Salvador señalaron que este tema estaba incluido en documentos en donde se daban lineamientos para ivestigación de enfermedades emergentes, estando ausente algún documento específico. En cuanto al registro de la investigaciones realizadas en Covid-19 o información del número de investigaciones realizadas, Costa Rica identificó 12 estudios, Honduras 43 y Panamá informó que lleva un registro en el Comité Nacional de Bioética de la Investigación (CNBI) con 111 estudios de Covid-19 hasta marzo de 2021. El resto de los países no disponen de registros actualizados. Con relación a las Medidas tomadas como respuesta para asegurar revisión ética rigurosa y acelerada, Costa Rica destacó que el 5 de agosto después de declarada la 
pandemia se publicó el documento "Comunicado 2 del CONIS". Por su parte Panamá había trabajado previo a la declaración de la Pandemia en el país, un procedimiento operativo para la aprobación de estudios en situaciones de desastres y brotes de enfermedades de acuerdo con la pauta 20 del Consejo de las Organizaciones Internacionales de las Ciencias Médicas (CIOMS), y el 13 de abril de 2020 el Ministerio de Salud emitió la Resolución 373 que centraliza la revisión ética en el CNBI. El resto de los países no presentaron documentos que sustentaran las medidas. Costa Rica y Panamá tienen una base regulatoria sólida, aunque en temas de gobernanza se requiere que la ética sea reconocida como parte central del proceso de investigación por las autoridades y se pondere la importancia de aumentar la calidad de la investigación. Con relación al desarrollo de capacidades el estudio demostró que hay necesidades de capacitación del recurso humano, y necesidad de incrementar la oferta académica de programas regionales de posgrado en ética en investigación que pudieran servir como certificación para los miembros de los comités de ética y propiciar programas de educación continua en bioética. Finalmente se destacó que hay que darle atención a la asignación de recursos financieros y tecnológicos para los comités de ética. Se hizo énfasis en que el estatus ad honorem de la mayoría de los miembros de los comités de éticas, pese a los esfuerzos de adaptación del proceso de revisión, ha sido una de las causas de retraso en la revisión, modificación y aprobación de los proyectos de investigación.

Cuadro №1

Resultados Taller №2 GoÉtica

\begin{tabular}{|c|c|c|c|c|c|c|}
\hline & Costa Rica & El Salvador & Guatemala & Honduras & Panamá & República Dominicana \\
\hline $\begin{array}{l}\text { Existencia de } \\
\text { Documento escrito } \\
\text { con la prioridad de } \\
\text { Investigación para } \\
\text { Covid-19 } \\
\end{array}$ & No & $\begin{array}{l}\text { No específico. Incluido en } \\
\text { enfermedades } \\
\text { emergentes }\end{array}$ & No & No & $\begin{array}{l}\text { No específico. Incluido } \\
\text { en enfermedades } \\
\text { emergentes }\end{array}$ & No \\
\hline $\begin{array}{l}\text { Procedimiento } \\
\text { operativo para dar } \\
\text { respuesta a la } \\
\text { Pandemia }\end{array}$ & $\begin{array}{l}\text { Si. Después de } \\
\text { iniciada la } \\
\text { Pandemia }\end{array}$ & $\begin{array}{l}\text { Si } \\
\text { Acta de sesión del CNEIS }\end{array}$ & $\begin{array}{l}\text { Si. } \\
\text { Enlace no } \\
\text { disponible } \\
\text { públicamente }\end{array}$ & No & $\begin{array}{l}\text { Procedimiento } \\
\text { Operativo PO-026 }\end{array}$ & No \\
\hline $\begin{array}{l}\text { Registro de } \\
\text { Investigaciones } \\
\text { realizadas }\end{array}$ & $\begin{array}{l}\text { Se identificaron } 12 \\
\text { estudios. Registro } \\
\text { oficial no } \\
\text { actualizado }\end{array}$ & $\begin{array}{l}\text { Registro oficial no } \\
\text { actualizado }\end{array}$ & $\begin{array}{l}\text { Registro no } \\
\text { disponible } \\
\text { públicamente }\end{array}$ & $\begin{array}{l}\text { No existe } \\
\text { registro. Se } \\
\text { identificaron } \\
43\end{array}$ & $\begin{array}{l}111 \text { estudios } \\
\text { registrados hasta } \\
\text { marzo de } 2021 . \\
\text { A junio } 129 .\end{array}$ & Desconocido \\
\hline $\begin{array}{l}\text { Medidas tomadas } \\
\text { como respuesta para } \\
\text { asegurar la revisión } \\
\text { ética rigurosa y } \\
\text { acelerada }\end{array}$ & $\begin{array}{l}\text { Si. Comunicado } 2 \\
\text { del CONIS }\end{array}$ & $\begin{array}{l}\text { Dar prioridad a las } \\
\text { investigaciones } \\
\text { relacionadas a Covid-19. } \\
\text { Establecer tiempos } \\
\text { inmediatos Definir } \\
\text { comisión para revisión } \\
\text { expedita de protocolos } \\
\text { Covid-1 }\end{array}$ & & & $\begin{array}{l}\text { POE 026. Resolución } \\
373 \text { de } 13 \text { de abril de } \\
2020 \text {. } \\
\text { Centralización de la } \\
\text { revsión en el CNBI y } \\
\text { respuesta en horas }\end{array}$ & NO \\
\hline $\begin{array}{l}\text { Gobernanza y Apoyo } \\
\text { Institucional }\end{array}$ & Ley 9234 & & & & Ley 84 de 2019 & \\
\hline
\end{tabular}

Particularmente en Panamá, se hizo necesaria una evaluación de la experiencia, porque la centralización de la revisión ética en el CNBI y la respuesta en horas, hizo que el sistema se congestionara sobre todo en el trabajo de la secretaría técnica. Como resultado de esta evaluación se tomaron las siguientes decisiones: derivar los estudios académicos, observacionales, epidemiológicos a los comités de bioética institucionales, 
solictar el apoyo de los secretarios técnicos de los comités de bioética acreditados para atender la corrrespondencia del CNBI, dar cortesía de sala a los investigadores de ensayos clínicos antes del ingreso formal del estudio para ir resolviendo dudas a manera de preevaluaciones, consulta a expertos, nombramioento de revisores externos. En consecuencia hubo incumplimiento del plan de capacitacitaciones en el 2020 y la postergación del uso de la plataforma proEthos. Entre los logros podemos mencionar: la migración a la virtualidad para la evaluación ética, procedimientos operativos para la re acreditación semi presencial de comités institucionales, implementación de Procedimientos Operativos Estandarizados, para situaciones de emergencia y brotes de enfermedades, colaboración internacional para compartir experiencias y fortalecer de la gobernanza en ética de la investigación en la región Centroamericana y República Dominicana. En conclusión Panamá ha dado pasos importantes en el desarrollo de la Bioética de la Investigación, en la región centroamericana es el país con mayor avance en este tema. Tenemos una Ley, un registro nacional de la investigación $y$, aunque nos falta desarrollar otros aspectos, hemos avanzado yy positivamente. Nuestra Ley servirá de marco de referencia para la región, particularmente República Dominicana que ha consultado su texto.

\section{CONCLUSIONES}

La Dra. Ana Sánchez como moderadora concluye con los puntos mas importantes de la mesa. La Pandemia puso en evidencia las desigualdades en todos los niveles. Por otra parte, la revisión acelerada no debe ser nunca un atajo a la rigurosidad de la evaluación. También el tema de tratamientos no probados ha sido un tema relevante en el que hace falta más información al público y a los médicos tratantes. Resaltamos la importancia que ha habido en los registros de investigación y en la publicación de resultados negativos, en esta emergencia sanitaria se ha visto lo relevante que ha sido todo ese proceso. Una lección aprendida, es que debemos mantener los horarios laborales para que no se produzca el agotamiento, dado que lo que se pensó era una carrera de velocidad se convirtió en una carrera de resistencia. Por otra parte, es importante la colaboración regional a través de la OPS, pero también la colaboración horizontal porque nos permite ver nuestras debilidades, y nuestras fortalezas. En estos últimos años Panamá ha evolucionado mucho en el sentido de registro, hace 8 o 9 años solo se revisaban ensayos clínicos, ahora estamos haciendo evaluaciones de estudios de investigación con seres humanos, ponderando la importancia de la protección de la población participante, tomando en cuenta que por más que la intervención física sobre la persona sea muy limitada los estudios pueden no ser inocuos. Ha habido un aprendizaje de los comités, pero también de los investigadores, en un inicio pensaban que era absurdo requerir evaluación ética cuando no era un ensayo clínico, han ido aprendido que tener una revisión ética los respalda, los protege y protege sobre todo a 
la población que participa en los estudios y que a veces una fuga de información puede ser mas dañina que una inyección mal puesta.

\section{BIBLIOGRAFÍA}

[1] OPS ¿Cómo promover la transparencia en la investigación? Acciones para las autoridades nacionales durante la pandemia de COVID-19. 2020

[2] https://iris.paho.org/bitstream/handle/10665.2/52767/OPSHSSBIOCOVID-19200034 por. pdf?sequence $=1$ \&isAllowed $=\mathbf{y}$

[3] OPS. Modelo y Pautas operativas para la revisión y supervisión éticas de las investigaciones relacionadas con COVID-19. 2020

[4] https://www.paho.org/es/documentos/modelo-pautas-operativas-para-revision-supervision-eticas-investigacionesrelacionadas

[5] OPS. Orientación y estrategias para agilizar la revisión y supervisión éticas de investigaiones relacionadas con COVID-19. 2020

[6] https://iris.paho.org/handle/10665.2/52090

[7] OPS. "Uso de emergencia de intervenciones no probadas y fuera del ámbito de la investigación: orientación ética para la pandemia de Covid-19", 2021

[8] https://iris.paho.org/handle/10665.2/52430

[9] Cabré, P. LI.; Casado M. Recomendaciones para la toma de decisiones éticas sobre el acceso de pacientes a unidades de cuidados especiales en situaciones de pandemia .Observatorio de Bioética y Derecho UB. 2020. http://hdl.handle.net/2445/154297

\section{Autorización y Licencia CC}

Los autores autorizan a APANAC XVIII a publicar el artículo en las actas de la conferencia en Acceso Abierto (Open Access) en diversos formatos digitales (PDF, HTML, EPUB) e integrarlos en diversas plataformas online como repositorios y bases de datos bajo la licencia CC: Attribution-NonCommercial-ShareAlike 4.0 International (CC BY-NC-SA 4.0) https://creativecommons.org/ licenses/by-nc-sa/4.0/.

Ni APANAC XVIII ni los editores son responsables ni del contenido ni de las implicaciones de lo expresado en el artículo. 